\title{
Carta Editorial
}

Sandro Dutra e Silva ${ }^{1}$, Marina Miraglia ${ }^{2}$, Antonio Ortega Santos ${ }^{3}$, Chiara Olivieri ${ }^{4}$, Martha Micheline C. Olvera ${ }^{5}$, Pablo Corral Broto ${ }^{6}$

La edición correspondiente al número 1 del volumen 9 (2019) de HALAC: Historia Ambiental Latinoamericana y Caribeña presenta un conjunto de 7 (siete) artículos, 1 (un) ensayo, 3 (tres) reseñas que componen el dossié "Territorios Comunes Herramientas Frente Al Capitaloceno". Este dossié presenta un conjunto diverso de abordajes, temáticas, metodologías y discusiones que refuerzan el escenario complejo y dinámico del conocimiento ambiental en su diálogo histórico. La primera edición de 2019 contiene importantes contribuciones de centros de investigación y Universidades de España, Argentina, Chile, Brasil, Francia y Estados Unidos, reflejando la diversidad geográfica de los abordajes histórico-ambientales aquí presentes.

\footnotetext{
1 Doctor en Historia por la Universidad de Brasilia. Coordinación Editorial de la Revista Fronteiras: Journal of Social, Technological and Environmental Science. Docente en la Universidad Estadual de Goiás - UEG y en el Centro Universitario de Anápolis - UniEVANGÉLICA . Brasil. Email: sandrodutr@hotmail.com

2 Doctora en Filosofia y Letras con Orientación en Geografía, Facultad de Filosofia y Letras de la Universidad de Buenos Aires. Docente en la Universidad Nacional de General Sarmiento y Universidad Nacional de Quilmes, Argentina. Email: marina.miraglia@gmail.com

${ }^{3}$ Doctor en Historia Contemporánea Universidad de Granada y Profesor Titular Historia Contemporánea, Facultad Filosofía y Letras, Universidad de Granada. Email: aortegas@ugr.es

${ }^{4}$ Becaria Investigación FPU, Departamento Historia Contemporánea/Instituto de Investigación sobre Migraciones, Universidad de Granada, email: olivieric@ugr.es

${ }^{5}$ Doctora en Historia por la École des Hautes Études en Sciences Sociales, en París, Francia Profesora Investigadora de tiempo completo Titular C, Universidad Autónoma de Baja California Sur. Email marthamichelinecarino@gmail.com

${ }^{6}$ Doctor en Historia por la Universidad de Granada. Maître de conférences en Civilisation Espagnole à l'Université de La Réunion (Département d'Études Hispaniques). Email: pablo.corral-broto@univ-reunion.fr
} 
Desde hace tiempo, la Red Universitaria Española de Historia Ambiental (RUEDHA), y desde las tareas y proyectos empreendidos y liderados por del Grupo HUM952 STAND (Universidad de Granada) se viene desarrollando encuentros bianuales con el fin de promover un diálogo continuado con investigadores del contexto de la Península Ibérica al respecto de la agenda de trabajo de la Historia SocioAmbiental. Tras el último encuentro en Granada en el 2018, se convocó a los investigadores que desde muchas áreas científico académicas, se sienten llamados a estudiar y analizar la relación que las sociedades humanas y la naturaleza han tenido a lo largo de la Historia. Este dossié supone una puesta al día de las líneas emergente en el campo de la historia ambiental española en sus diálogos con América alrededor de una serie de ejes temáticos, desplegados por los grupos líderes en estos campos de investigación.

Con todo ello se dispone de un panorama sobre territorios comunes de conocimiento que enlazan y trazan puentes de colaboración en el contexto americano y español como punto de encuentro a la hora de la renovación epistémica. De este modo, y considerando la gran contribución de los autores mencionados, es con mucho agrado que se presenta el dossier "Territorios Comunes Herramientas Frente Al Capitaloceno".

En el primer artículo, el investigador Pablo Saralegui Díez, de la Universidad Pablo de Olavide de España, argumenta que el metabolismo social resulta una herramienta integral para analizar las transiciones históricas alimentarias por un lado, como las propuestas contestatarias al Sistema Agroalimentario Global en el que nos encontramos, por el otro. No obstante, se han desarrollado trabajos desde una perspectiva biofísica e histórica, siendo necesario analizar las nuevas cadenas agroalimentarias conformadas por movimientos sociales alimentarios, tanto por su actualidad como por la necesidad de contribuir a mejorar su funcionamiento. En esta contribución se pretende formular una 
categorización de las distintas cadenas agroalimentarias (agro-) ecológicas que funcionan en la ciudad de Madrid, integrando para ello una visión sobre el trabajo colectivo a lo largo de la cadena corta, y como pilar fundamental de su estructura y mantenimiento a lo largo del tiempo.

Jesús Ángel Redondo Cardeñoso, investigador de la Universidad de Valladolid, España, en su artículo "Resistencias cotidianas a la privatización de recursos naturales en Portugal a inicios del siglo XX", analiza la caza furtiva como forma de resistencia mediante la cual las comunidades rurales sortearon la legislación que restringía el libre usufructo de los recursos cinegéticos del entorno natural. Para ello, tomó como marco de estudio la región portuguesa del Alentejo entre los años 1910 y 1917. El texto se divide en tres partes: primero, se estudia la legislación sobre caza; segundo, se analiza la extensión de la caza ilegal en la región a través de la información recogida en periódicos regionales y documentación del gobierno civil y, tercero, se profundiza en el análisis de la naturaleza de la caza furtiva por medio de documentación judicial de ámbito local. La principal conclusión es que, a pesar de la legislación restrictiva, la caza furtiva fue una práctica generalizada en la región, realizada principalmente por individuos de las clases populares para obtener recursos económicos complementarios en épocas de desempleo.

"Iniciativa para la Integración Regional Sudamericana (IIRSACOSIPLAN): neoextractivismo y emergencia de Brasil y China en América Latina", escrito por Helios Escalante Moreno de la Universidad de Granada, analiza la inserción de América Latina en la economía mundial, la cual se ha caracterizado históricamente por la extracción de recursos naturales y materias primas dirigidos a los países centrales. Recientemente el relativo declive de la hegemonía de Estados Unidos ha permitido dejar espacio para la emergencia de otras fuerzas, entre ellas se destacan Brasil como potencia regional y China como potencia internacional, necesitados de recursos 
naturales para el crecimiento de sus economías. Todas estas tendencias se reflejan en la articulación del megaproyecto IIRSA-COSIPLAN (Iniciativa para la Integración Regional Sudamericana), basado en las construcciones de infraestructuras, especialmente corredores de transporte y energéticas, destinadas a facilitar dicha extracción y exportación de recursos. La historia de este megaproyecto está vinculada con los cambios políticos vividos en América Latina en la primera década del siglo XXI y los distintos procesos de integración regional, marcados por el paso de gobiernos abiertamente neoliberales a los llamados gobiernos progresistas.

Adlina Codina Canet, de la Universidad Carlos III de Madrid, en conjunto con Elena Diaz Casero y Eduardo Saiz Riquelme, presentan un artículo que se aproxima a una nota científica, donde relatan la creación de un archivo digital del movimiento ecologista español y de los conflictos ambientales en los que ha participado desde su aparición en la década de los años 70. En "Elaboración de un archivo de memoria del movimiento ecologista a partir de los fondos de la confederación ecologistas en acción" los investigadores afirman que el proyecto es un intento de construir bases para el desarrollo de una historia ambiental en su país, dando libre acceso de los fondos documentales, que unos de los principales protagonistas conserva. El proyecto se inició en mayo de 2017 y entre los logros conseguidos se halla la difusión del proyecto en diferentes foros y artículos. Se ha creado una plataforma digital, y han sido agregados cerca de 1.200 documentos. El estudio aporta un análisis de la grupalidad del movimiento ecologista en España en las décadas de los años setenta, ochenta y noventa.

El trabajo "Reconstruyendo la degradación ambiental del Lago Vichuquén, Región del Maule, Chile, mediante el uso de registros históricosdocumentales", fue realizado por los investigadores Pablo César Pedreros Troncoso, Fernando Torrejón, Denisse Álvarez, Roberto Urrutia de las Universidades de Concepción y Santo Tomás de Chile, respectivamente. 
Mediante un análisis histórico-documental se reconstruyeron las condiciones ambientales del lago Vichuquén y su entorno entre los siglos XIX-XXI. Los resultados obtenidos permitieron determinar transformaciones del paisaje natural como consecuencia de la actividad humana. Se pudo establecer que hasta fines del siglo XIX la intervención ambiental habría sido moderada, momento en que comenzó a intensificarse notoriamente debido a la explotación mercantil de recursos naturales y al cambio de uso de suelo. Desde mediados del siglo XX, la ocupación ribereña del lago con fines turísticos-recreacionales incrementó severamente la degradación ambiental del ecosistema léntico, aumentando su nivel de trofismo y se verificó además la importante incidencia de algunos fenómenos naturales en la dinámica del complejo lacustre de Vichuquén.

Ana Marcela França, del Centro de Estudios de la Argentina Rural UNQ, analiza en su artículo titulado "Patrimônio e paisagem: a escrita da história ambiental do Parque Costero del Sur (Punta Indio, Argentina)" la relación entre naturaleza y sociedad impresa en los paisajes del Parque Costero del Sur y alrededores. Por medio de la historia ambiental, se busca valorar la memoria de la zona a través de su paisaje, incluyendo el reconocimiento de especies vegetales nativa y exótica, la introducción de animales domesticables y su complejidad, así como la valoración de bienes materiales e inmateriales que narran la historia de esa parte de la pampa deprimida. La investigación es parte de un proyecto que busca conectar los paisajes relacionados al parque con el archivo del Museo histórico del Partido de Punta Indio.

El último artículo tiene como título "Cambios y permanencias en la producción de yerba mate: Un estudio desde la historia ambiental en Misiones (Argentina)". Escrito por la investigadora María Cecilia Gallero, del Instituto de Estudios Sociales y Humanos de la Universidad Nacional de Misiones y el Consejo Nacional de Investigaciones Científicas y Técnicas 
(CONICET), Argentina. La autora investiga la transformación que sufrió el ambiente de Misiones a partir de la implementación de los yerbales cultivados a principios del siglo XX. Para ello, tomó como estudio de caso la Cooperativa de "Productores de Yerba Mate de Santo Pipó S.C.L.", pues además de ser la primera cooperativa especializada, fue fundada por suizos que migraron con el fin de dedicarse al cultivo de la yerba mate. Estudiar la producción de la yerba mate a través del prisma de la historia ambiental permite explicar el impacto provocado por los seres humanos en el territorio misionero. En este sentido, la llegada de inmigrantes suizos tuvo especial repercusión en la mudanza de una economía extractiva a una productiva, pues contribuyeron a arraigarla como cultivo intensivo y explican la razón por la cual, la Argentina sea el principal productor mundial de yerba mate, seguido por Brasil y Paraguay.

El historiador ambiental John McNeil, de la Universidad de Georgetown (Washington, DC) presenta un importante ensayo sobre el Antropoceno en el contexto de la Historia Ambiental en los Estados Unidos de América (EUA). El texto está dividido en tres ejes que se integran: la tradición de la historia ambiental practicada en los últimos 40 años en los EUA; el impacto sobre esas tradiciones del concepto de Antropoceno y finalmente, la situación actual en los EUA en relación a la historia ambiental.

En esta edición de HALAC, se presentan también tres reseñas de obras publicadas en Brasil y Estados Unidos. En "Frontier and Western History in Central Brazil", el renombrado historiador Donald Worster, hace un análisis de la relación entre los estudios de frontera en los Estados Unidos y Canadá, a partir de Western History y la historia de la frontera en Brasil, por medio de la obra de Dutra e Silva, "No Oeste, a Terra e o Céu: a expansão da fronteira agrícola no Brasil Central" (Rio de Janeiro: Mauad X, 2017). 
Marcio Henrique Bertazi, de la Universidad Federal de São Carlos, hace un importante análisis de la obra de la socióloga holandesa Saskia Sassen, "Expulsões: brutalidade e complexidade na economia global (Rio de Janeiro: Paz e Terra, 2016). Con la reseña titulada "Geopolíticas da destruição: deslocamentos humanos e ambientais" argumenta que, la obra evidencia una serie de fenómenos que explican la brutalidad y la complejidad de la nueva economía global, responsable directa de las diversas formas de expulsión, inclusive ambientales.

Viridiana Hernández Fernández (Universidad de California, Davis) presenta, en su "Revolución y reparto agrario en la Ciudad de México" una reseña de la obra de Matthew Vitz titulada "A City on a Lake: Urban Political Ecology and the Growth of Mexico City (Durham: Duke University Press, 2018). Según Fernández, la obra de Vitz presenta, por medio de una rica documentación, un análisis de los procesos históricos del sorprendente crecimiento experimentado por la Ciudad de México entre los años de 1890 hasta mediados del siglo XX, envolviendo aspectos amplios como las cuestiones ambientales, los humanos y los no humanos.

La última reseña, titulada "Geopolíticas de la destrucción: desplazamientos humanos y ambientales", Marcio Henrique Bertazi, de la Universidad de São Paulo, presenta la relación entre formas extremas de pobreza y violencia seguido del aumento en la escala de destrucción de la biosfera, a partir de la obra de la socióloga holandesa Saskia Sassen.

El equipo editorial de HALAC, desea que estas contribuciones reciban amplia visibilidad y contribuyan al desarrollo de los debates en historia ambiental latinoamericana, en todos los espacios académicos, científicos, políticos y sociales que se interesen en estos temas historiográficos. 